INPLASY

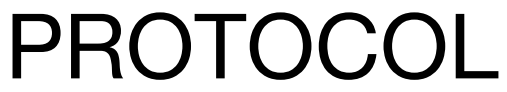

To cite: Han et al. The comparative efficacy and safety of acupuncture for mild and moderate Alzheimer's disease:A systematic review and network meta-analysis. Inplasy protocol 2021110014. doi:

10.37766/inplasy2021.11.0014

Received: 04 November 2021

Published: 04 November 2021

Corresponding author:

Nana Han

1016778077@qq.com

Author Affiliation:

Beijing University of Chinese Medicine

Support: 2019YFC1712100.

Review Stage at time of this submission: Preliminary searches.

Conflicts of interest: None declared.

\section{The comparative efficacy and safety of acupuncture for mild and moderate Alzheimer's disease: A systematic review and network meta-analysis}

\author{
Han, N1; Fang, Y2; Zhao, G33 Ji,B4.
}

Review question / Objective: According to the current randomized clinical trials (RCT) of acupuncture therapy for Alzheimer's disease (AD), to evaluate their methodology, the quality of evidence and the report are evaluated and summarize evidence of important outcomes of randomized clinical trials. We aim to provide accurate clinical decisionmaking for acupuncture treatment of Alzheimer's disease. Condition being studied: According to the current randomized clinical trials (RCT) of acupuncture therapy for Alzheimer's disease (AD), to evaluate their methodology, the quality of evidence and the report are evaluated and summarize evidence of important outcomes of randomized clinical trials. We aim to provide accurate clinical decision-making for acupuncture treatment of Alzheimer's disease.

INPLASY registration number: This protocol was registered with the International Platform of Registered Systematic Review and Meta-Analysis Protocols (INPLASY) on 04 November 2021 and was last updated on 04 November 2021 (registration number INPLASY2021110014).

\section{INTRODUCTION}

Review question / Objective: According to the current randomized clinical trials (RCT) of acupuncture therapy for Alzheimer's disease (AD), to evaluate their methodology, the quality of evidence and the report are evaluated and summarize evidence of important outcomes of randomized clinical trials. We aim to provide accurate clinical decision-making for acupuncture treatment of Alzheimer's disease.

Condition being studied: According to the current randomized clinical trials (RCT) of acupuncture therapy for Alzheimer's disease (AD), to evaluate their 
methodology, the quality of evidence and the report are evaluated and summarize evidence of important outcomes of randomized clinical trials. We aim to provide accurate clinical decision-making for acupuncture treatment of Alzheimer's disease.

\section{METHODS}

Search strategy: Three English and four Chinese medical databases, including PubMed, The Cochrane Library, EMBASE, the China National Knowledge Infrastructure (CNKI [Chinese version]), Wanfang Data (Chinese version), CQVIP (Chinese version), and SionMed (Chinese version) were electronically searched from inception to September 2021. Only studies published in Chinese or English were included in this systematic review and network meta-analysis. According to the "PICOS" principle to formulate the search strategy, we used search terms including: "Alzheimer disease","Transcranial Direct Current Stimulation" "acupuncture", "acupuncture therapy", "Donepezil", "Galantamine", "Rivastigmine", "Memantine", "Huperzine A", "Ginkgo Biloba", "Cognitive Behavioral Therapy".

Participant or population: Participants were definitely diagnosed as mild or moderate Alzheimer's disease,including but not limited to the following diagnostic criteria:the criteria of the Diagnostic and Statistical Manual of Mental Disorders (DSM), the National Institute of Neurological and Communicative Disorders and Stroke and the $A D$ and Related Disorders Association (NINCDS-ADRDA) criteria,The National Institute on Aging and the Alzheimer's Association (NIA-AA) developed research criteria and the operational criteria for the diagnosis of $A D$ (OCDAD).Patients were irrespective of gender, ethnicity or nationality.

Intervention: Acupuncture therapy.

Comparator: Donepezil, Galantamine, Rivastigmine, Memantine, Huperzine $A$, Ginkgo, Transcranial direct current stimulation, Cognitive Therapy, placebo/ blank.

Study designs to be included: Randomized control trials.

Eligibility criteria: Published randomized control trials which were reported in Chinese or English, and meet the "PICOS", will be considered for inclusion in this study.

Information sources: PubMed, Cochrane Library, Embase, Chinese Biomedical Literature Database (CBM), China National Knowledge Infrastructure (CNKI), Wanfang Data and Chongqing VIP (CQVIP).

Main outcome(s): At least one of the following outcome assessments: (1)Minic Menial statc Examination; (2) Alzheimer's Disease Assessment Scale-Cognitive section (ADAS-Cog); (3) Activity of Daily Living(ADL); (4)Neuropsychiatric Inventory (NPI); (5)adverse reaction.

Quality assessment / Risk of bias analysis: Risk of bias equality assessment Assess the quality of evidence in the included reviews using GRADE. Assess the methodological quality of the included reviews using Cochrane ROB. Assess the quality of report in the included reviews using CONSORT.

Strategy of data synthesis: We estimated summary odds ratios (ORs) for dichotomous outcomes and standardised mean differences (SMD, Cohen'sd) for continuous outcomes using pairwise and network meta-analysis. Risk ratio(RR) for both fixed and random effects models (weighting by inverse of variance) will be used. A continuity correction will also be used for cells with zero values. Betweenstudy heterogeneity will be assessed using the $t$, $\times 2$ (Cochran $Q$ ) and $I^{2}$ statistics. According to the Cochrane handbook, the 2 will be considered non-important $(60 \%)$. Results will be assessed using forest plots and presented as RRs for the main outcome and secondary outcomes. An influence analysis will be performed to ascertain the results of the meta-analysis 
by excluding each of the individual studies. Statistical analysis will be conducted using STATA software for Windows v16.0.

Subgroup analysis: State any planned investigation of 'subgroups'.Be clear and specific about which type of study or participant will be included in each group or covariate investigated. State the planned analytic approach. To be determined based on the literature retrieved.

Sensitivity analysis: Publication bias will be assessed by a funnel plot for meta-analysis and quantified by the Egger method.

Country(ies) involved: China.

Keywords: Alzheimer's disease; acupuncture; PRISMA; GRADE; network meta-analysis.

Contributions of each author:

Author 1 - Nana Han.

Email: 1016778077@qq.com

Author 2 - Yang Fang.

Email: yangfang113@foxmail.com

Author 3 - Guozhen Zhao.

Author 4 - Bo Ji. 